\title{
IMAGE BASED RECOGNITION - RECENT CHALLENGES AND SOLUTIONS ILLUSTRATED ON APPLICATIONS
}

\author{
Daniel Garten ${ }^{1}$, Katharina Anding ${ }^{2}$ and Steffen Lerm ${ }^{2}$ \\ ${ }^{1}$ Society for Production Engineering and Development, Schmalkalden, Germany \\ ${ }^{2}$ Ilmenau University of Technology, Department of Quality Assurance and Industrial \\ Image Processing, Ilmenau, Germany
}

\begin{abstract}
In this paper, problems and solutions for the automatic recognition of miscellaneous materials, especially bulk materials are discussed. The fact that many materials, especially natural materials, have a strong phenotypic variability resulting in high intra-class and low inter-class variability of the calculated features poses a complex recognition problem. The recognition of components of a wheat sample or the classification of mineral aggregates serves as an example to demonstrate different aspects in segmentation, feature extraction, classifier design and complexity assessment. We present a technique for the segmentation of highly overlapping and touching objects into single object images, a proposal for feature selection and classifier parameter optimization, as well as a method to visualise the complexity of a highdimensional recognition problem in a three-dimensional space. Every step of the pattern recognition process needs to be optimized carefully with special attention to the risk of overfitting. Modern processors and the application of field-programmable gate arrays as well as the outsourcing of processing steps to the graphic processing unit speed up the calculation and make real-time computation possible also for highly complex recognition problems such as the quality assurance of bulk materials.
\end{abstract}

\section{KEYWORDS}

Image Processing, Machine Learning, Complex Recognition Problems, Support Vector Machine, Feature Selection

\section{INTRODUCTION}

The development in microelectronics allows the use of modern but time-consuming algorithms that could be calculated in real-time because of the higher performance of current microprocessors. The prices of microprocessors are decreasing as a result of mass production. These facts make it possible to develop effective algorithms and to tackle problems in the field of image processing.

As a consequence of these technological advancements, new fields of application are emerging which could establish a new economic market. One of these new markets is the quality assurance of natural bulk goods like grain or stones with an image acquisition and processing system. Naturally, material-based specific problems appear on the high intra-class variability and the low inter-class variability and should be solved in an effective way.

For example, the consumption of grain is rising continuously. One reason is the growth of the world's population against the backdrop of the difficulty of permanently decreased cultivation areas due to climate changes. Food scandals increase the demand for high-quality food. 
Another commercial field for image processing systems is the recognition of mineral aggregates. The production of concrete, for example, poses a problem. Wrong concrete ingredients may reduce the life-time of the concrete and result in higher repair costs. Each kind of stone has individual chemical properties which influences the quality of the produced concrete. Certain components of aggregates can negatively affect the mechanical strength and durability of produced concrete or asphalt and also cause the destructive alkali silica reaction. Knowing the chemical decomposition process helps the expert to produce high-quality products, but the necessary chemical analysis of mineral aggregates is a very time-consuming process. Furthermore, chemical analysis cannot be used for analysing the quantity of mineral aggregates in concrete production. A promising method for solving this issue is the application of imageprocessing and recognition algorithms.

The given challenges require a complex dataset, representing all classes with known class labels (expert-sorted material). The recognition of natural bulk goods is complex because of high intraclass variability and a low inter-class variability of the objects, which mean a high phenotypic similarity between the different classes and the low specificity over all classes. Another problem is the mechanical separation of the material because of a wide range of shapes and specific weights. Hence, it is necessary to find a good segmentation routine in image processing, both in a mechanically separated view as well as a non-separated view.

\section{STATE-OF-THE-ART}

There are several color-sorting machines available on the market. Buehler is a global supplier of optical sorting machines for different applications, e. g. sorting of grain, coffee, oil seeds, tea and also non-food materials like plastics and recycling matter [1]. The color-sorting machines from Mogensen are applied for sorting limestone, marble, barite, salt as well as refractory material [2]. The color-sorting technology is designated for a very high operational capacity in the range of 5 25 tons per hour depending on the material. The discrimination between the classes to sort is done by simple and fast algorithms to calculate color and spectral features and, thus, major differences in color. A rough sorting - but no accurate quality analysis - is possible with these machines. The Acurum system was developed for an accurate quality analysis of Canadian wheat. The compact table top unit is able to classify and count the ingredients of a grain sample according to the Canadian official grain grading guide [3]. The system is not suitable for European wheat species. A similar system for the quality analysis of mineral aggregates is not available. In [4], the combination of classifiers for the complex task of rock image classification was investigated. The authors used MPEG-7 color and texture descriptors, Gabor features and other well-known image features within different combining strategies of k-nearest-neighbor classifiers. The dataset consists of 336 rock images divided into four classes by a human expert. The classes are not further described. They achieved recognition rates up to $81 \%$. In [5], it is demonstrated that hyperspectral fluorescence images can be very useful for discriminating between different minerals. Granite, talc, chlorite and magnesite were investigated. The required image acquisition system is very cost-intensive, so its application in small and medium-sized companies, especially testing organizations, is not expected. In our investigations, we focus on image based recognition by using machine learning techniques. Our aim is to demonstrate the possibilities with standard industrial cameras and illumination in the visible spectral range on two application areas: mineral and grain analysis.

\section{THE PROCESS OF TECHNICAL RECOGNITION}

The process of technical recognition consists of several steps. The aim of this process is to estimate the composition of bulk goods objects. Each type of bulk good has a specific material composition which needs to be analysed. Especially class objects with a negative influence 
urgently require such a recognition routine. Therefore, the percentage of each object class should be known in the given sample. Thus, each single object of the bulk goods has to be classified correctly. To classify single objects, computable features are necessary. For the calculation of features, each foreground pixel belonging to the object need to be found. This process is called segmentation. In this paper, the complete chain of image processing for technical recognition is presented.

The first step is the acquisition of suitable images. Although the experimental setup will not be described in this general chapter, it should be pointed out that the properties of the image acquisition system have a significant influence on the characteristics of the image data. Each component of the acquisition system should be adapted to the specific field of application. Information that is not part of the image data could not even be found by the best software algorithms. There are at least two possible settings to capture bulk goods.

One method, the so-called "separated view" entails a mechanical separation of each object of the bulk goods (Fig.1). The advantage of this method is the local separation of the objects. This makes it relatively easy to find single objects. However, the disadvantage of this method is the high effort needed for the mechanical separation device and its limited flow rate.

Another method is the so-called "compact view". Here, the objects are lying directly adjacent to each other in a continuum (Fig.2). The advantage should be clear; no mechanical separation units are needed. You need powerful algorithms to cut out each object from the acquired image of the camera. These algorithms have to be robust, and, furthermore, they have to be calculated in a justifiable time span.

The second step in the chain of image processing of the technical recognition is the image preprocessing. The image pre-processing serves to reduce annoyances like noise or methodological errors of the image acquisition system. In addition, image pre-processing can be used to normalise the image data. Pre-processing facilitates the next step in the chain of image processing, the segmentation.

The segmentation is the third step of the process of technical recognition. The challenge of the segmentation module is the determination of image foreground and uninteresting image background. In our field of application, the foreground contains the characteristics of the objects of bulk goods. To classify each single object of the bulk material, the segmentation has to estimate the coordinates of each object. In color image processing every pixel includes the color intensities of the red, green and blue channel. If the coordinates of the pixels and its corresponding color intensities of the acquired camera image of each object were known, all features of the objects could be calculated. This processing step is called feature extraction.

During feature extraction the discrete three-dimensional scalar field "colored image" is transformed into a vector of scalar numbers. This vector of scalar vectors is called a feature vector. The three dimensions of the colored input image are the dimensions in $\mathrm{X}$ and $\mathrm{Y}$ and the spectral channels of the image (red, green, blue). The feature vector includes properties of shape, color and texture.

Feature selection is the next step. It estimates the information content of each feature with regard to the specific class. The classification is the last step in the recognition process. The decision of which class the object belongs to is taken by a classifier. The classifier processes the input data (feature vector of an unknown object) and compares it with trained feature vectors. These trained feature vectors are called training dataset. The training dataset consists of the feature vector and its corresponding class label given by expert knowledge. 


\section{SEGMENTATION}

After the image is acquired and pre-processed, it has to be segmented. The aim of the segmentation is to estimate the coordinates of each object of bulk goods. There are different kinds of segmentation algorithms. They can be sub-divided into pixel-based, region-based, texturebased, edge-based and model-based segmentation algorithms. Furthermore, there are various possible hybrid methods. In this paper, a hybrid method will be described.

At this point, a distinction should be made between the segmentation of objects in "separated view" and "compact view".

\section{Segmentation of "separated view" images}

The objects of the bulk goods material have a dominant color. To build a suitable image acquisition system, the background should be chosen in a complementary color. If this condition is fulfilled, the segmentation problem should be fairly easy to solve. A pixel-based segmentation algorithm should be able to distinguish between background and foreground because each pixel should have a characteristic color. Either the pixel is similar to the dominant object color or to the complementary color of the pre-defined background.

The complex problem of the segmentation was solved by the mechanical separation unit, which allows it to keep the segmentation algorithm simple. This case of segmentation will not be described in this paper.

\section{Segmentation of "compact view" images}

A typical image of compact view is shown in Fig.2. In contrast to the separated view (Fig.1).

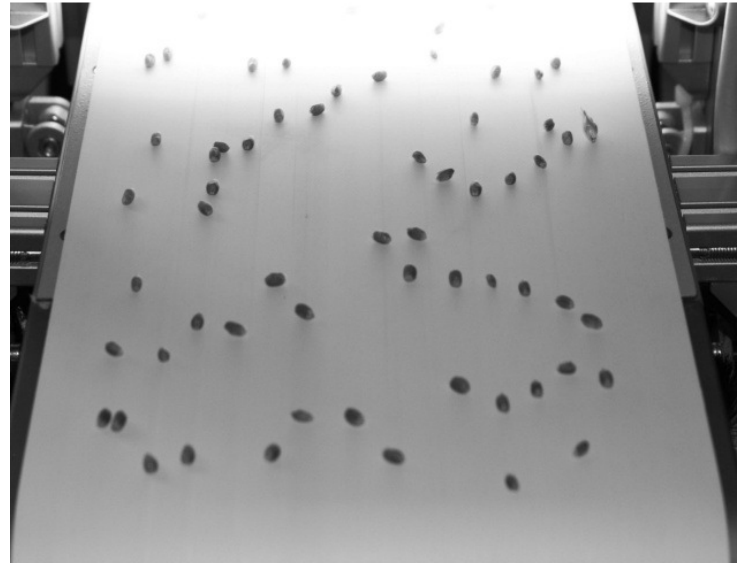

Fig. 1: Bulk goods in separated view on conveyer belt

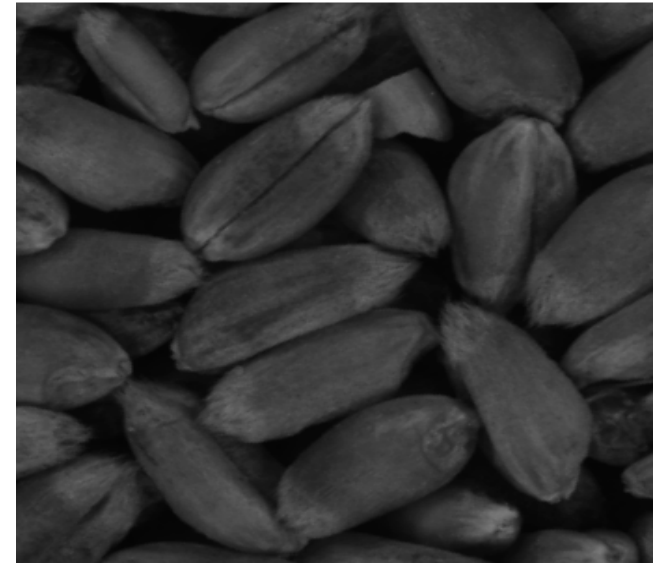

Fig. 2: Bulk goods in compact view (b/w transformed)

The original image is a RGB color image. Each pixel has 24 bits of information ( 8 bits per color channel). To solve the segmentation problem, the data has to be reduced from 24 bits to just one bit. This new binary image is called a binary cutting mask. "True" means that the pixel belongs to an object; "False" means that the pixel represents the background. The cutting mask, which has the same resolution as the original camera image, can be used to extract the pixel coordinates, and the coordinates can then be used to cut out the colored objects of the original camera image. 
In a first step, the input image data has to be analysed. In Fig.3, a false color image is shown. The RGB image was transformed to the YUV-color-space, and the Y-channel is displayed as a grayscale image. The most important fact is that the absolute intensity is not important to find an object. The information of the single object is within the relative signature of the intensities of directly neighbored pixels [6].

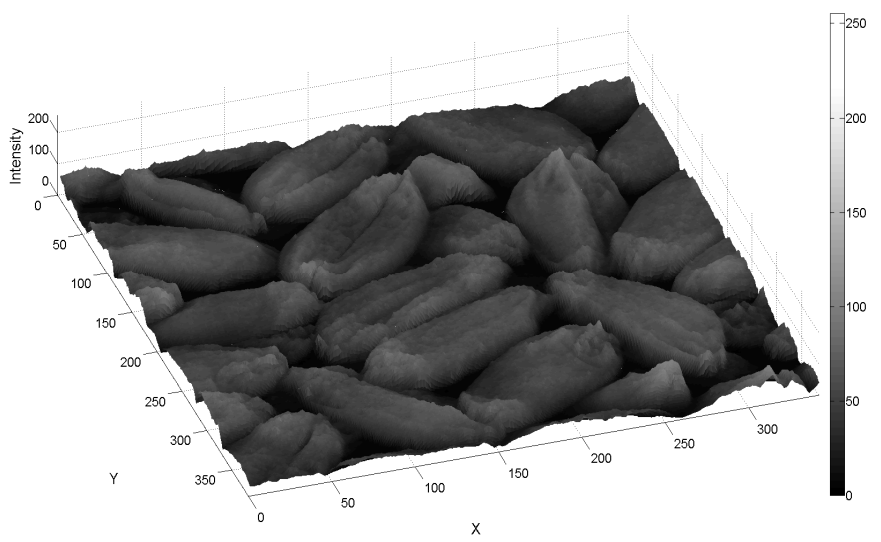

Fig. 3: False color image of a compact view image

To calculate the cutting mask a dynamic, threshold-based segmentation algorithm can be used. One challenge is to consider directly neighbored objects which are typical for a compact view of bulk goods. The edge information could be enhanced with the help of a filter.

Fig.4 displays the result of a color edge filter of the original RGB input image. The color edge image is a greyscale image. The input data for the calculation of the cutting mask are the original RGB color image and the color edge filtered image.

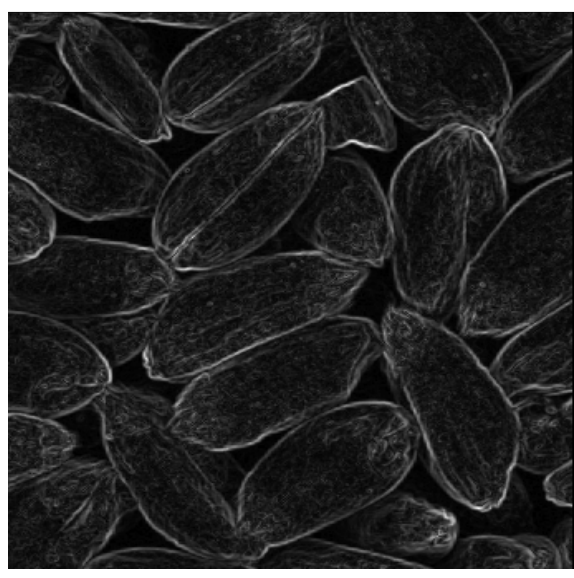

Fig. 4: Color edge image - compact view

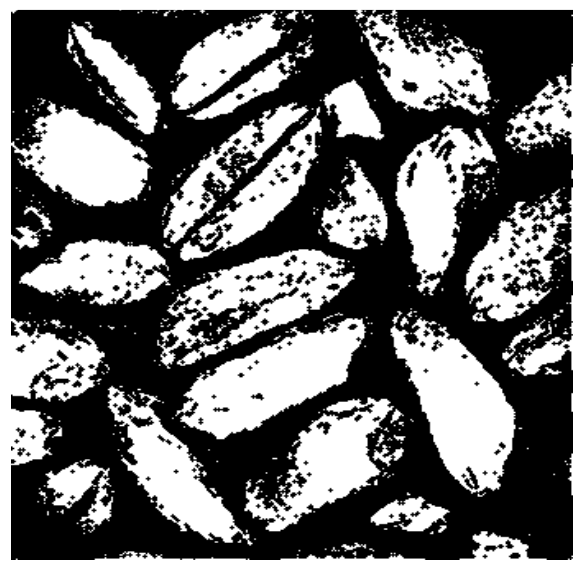

Fig. 5: Binary cutting mask - compact view

The quality of the binary cutting mask (Fig.5) is enhanced by a morphological closing. With a standard region-based segmentation process like a line coincidence algorithm, the binary cutting mask is transformed to a list of pixel coordinates. An overlay of the results of the first dynamic, threshold-based segmentation process is shown in Fig.6. The output data is a list of pixel coordinates. 


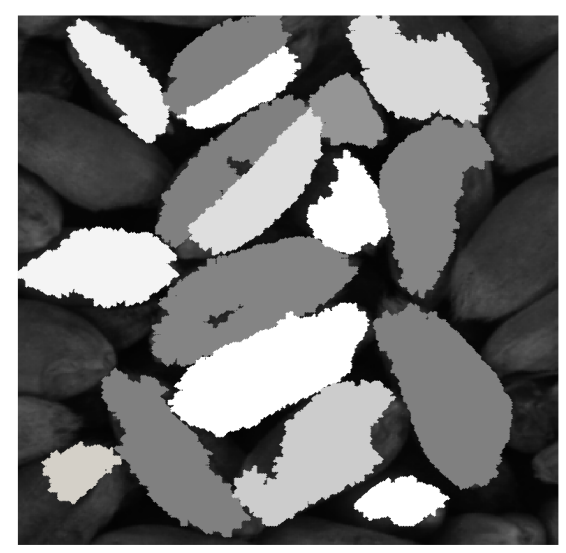

Fig. 6: Overlay of cutting mask in gray-scale level and original image

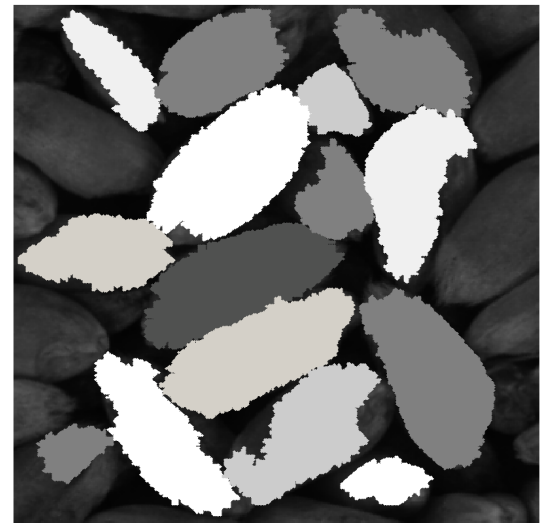

Fig. 7: Overlay of model-based post-segmentation of binary cutting mask and original image

This list of pixel coordinates is the input data for a second, model-based post-segmentation process. This second segmentation is done to consider a priori knowledge like average object size and special characteristics (e.g. furrow of grain kernels). Certain features of each region are calculated, such as the coordinates of the mass center, the area of the region and the slope of a regression straight line. If the features of each region fulfill the pre-defined values, then two or more regions could be fused. The difference of the results can be seen when comparing

Fig. 6 and Fig.7. Furthermore, the inner holes of the objects are filled. The post-segmentation enhances the segmentation performance and prevents failures in the classification process.

Finally, the pixel list can be used to cut out each single object. Fig. 8 shows the result of the cutting process. Digital images are always organised as rectangles in computer science. The segmented regions have irregular shapes, so they have to fill these irregular shapes to form rectangles. To avoid failures of the feature extraction, a specific color has to be used. In Fig.8, the background of the object was filled with white pixels to create a rectangular shape.

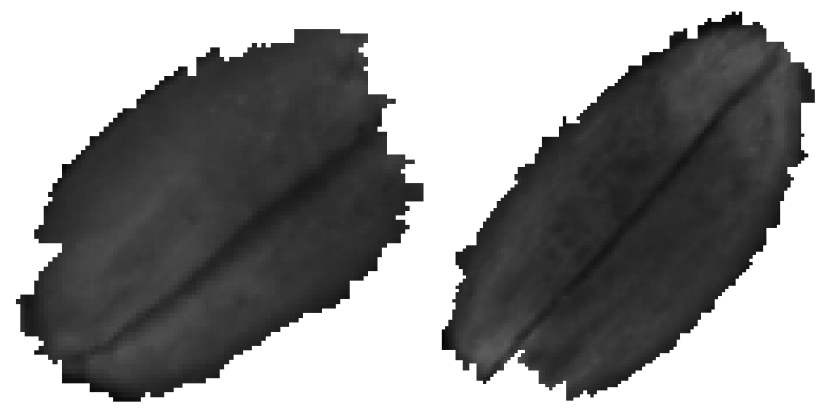

Fig. 8: Final segmentation result of object no. 6 and no. 7 (b/w transformed)

All single object images like in Fig. 8 are the input data for the next step in the chain of image processing of the technical recognition, the so-called feature extraction. 
Machine Learning and Applications: An International Journal (MLAIJ) Vol.3, No.3, September 2016

\section{FEATURE AND CLASSIFIER OPTIMIZATION}

\subsection{Feature set optimization}

Many classifiers suffer from the curse of dimensionality. Especially with natural materials it is often not clear which features carry relevant information. There is a variety of feature selection algorithms [7]. We have conducted an efficient, combined scheme of filter and wrapper. In the first step, the available dataset is randomly separated for feature evaluation (dataet 1 - DS1), classifier training (dataset 2 - DS2), and final testing (dataset 3 - DS3). In the next step, a score value for every feature is calculated on dataset 1 . The information gain value is recommended to score the features [8]. In the next step, all features are ordered according to their score value. Then, the number of the best ranked features used is successively reduced, and in every step the performance of a classifier trained with this feature set is estimated by cross-validation on the training set. This is done until a significant decrease in the classifier performance has been reached. For this, a McNemars significance test is used.

We have applied these algorithms to the problem of automatic quality assurance of wheat. In the system developed by us, a color line scan camera with 2048 pixels and a specialised separation device [11] was used to acquire single object images from every particle in a wheat sample of nearly $500 \mathrm{~g}$. It is necessary to choose a classifier for the relevance detection step which is fast in training and classification, and it is also sensitive to irrelevant features. Both requirements are fulfilled by the Naive bayes and a support vector machine (SVM) classifier. In this experiment, a bag-of-features with nearly 200 standard operators from the image processing library Halcon [9] as well as self-developed features based on texture information and gray value morphology [10] are used.

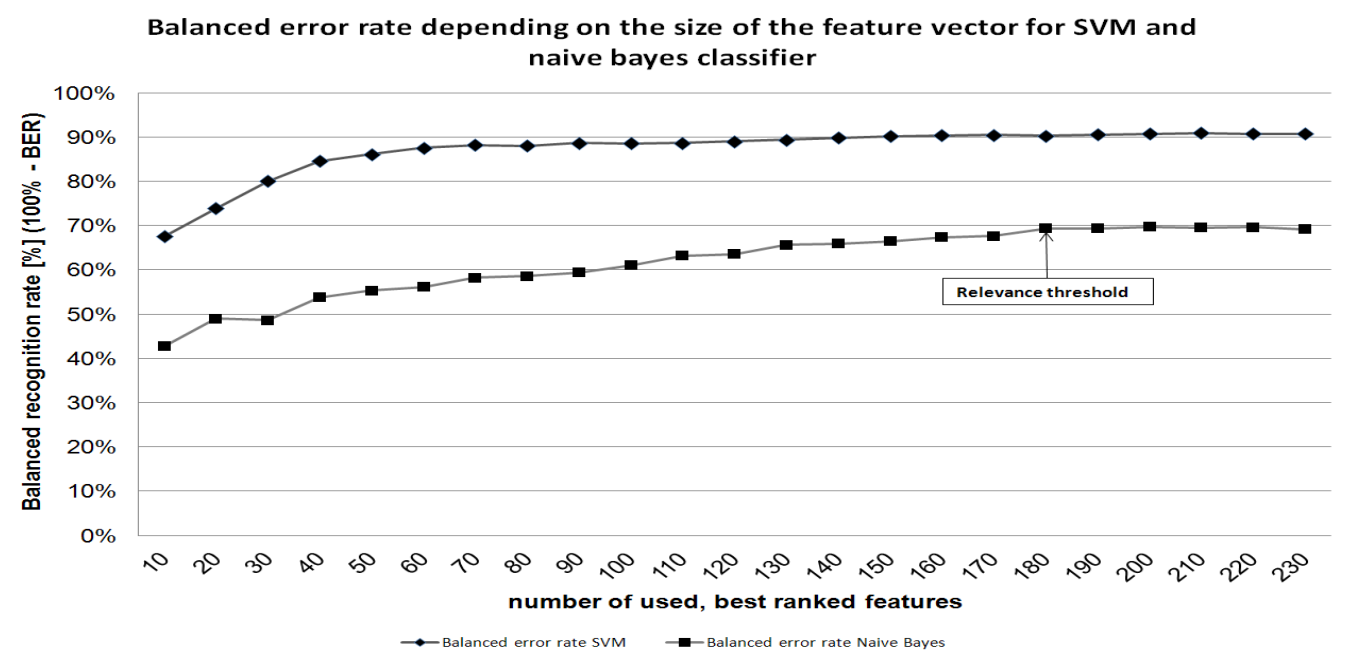

Fig. 9: Balanced recognition rate as a function of the number of best ranked features

The relevance threshold with a number of 180 features (see Fig.9) out of a total of 230 features could be estimated with the aforementioned processing scheme. The 10 worst ranked features were removed gradually. In every step, it has been checked if there was a significant decrease in the recognition rate in relation to the use of all possible features. The level of significance was chosen at $95 \%$ for the McNemar test. After reaching the threshold of 180 features in the feature set, further removing features results in a significant decrease in the balanced recognition rate. The characteristics of the recognition rates for the single classes are similar. The recognition rate 
Machine Learning and Applications: An International Journal (MLAIJ) Vol.3, No.3, September 2016

on the final test set shows that there is a significant improvement due to the introduction of problem-adapted image features and the reduction of the feature set to the most relevant features (Table 1).

Table 1: Results of the classification of grain on the test set

\begin{tabular}{|l|c|c|c|}
\hline \multicolumn{1}{|c|}{ class } & $\begin{array}{c}\text { recognition rate - feature set } \\
\text { with all standard operators }\end{array}$ & $\begin{array}{c}\text { recognition rate } \\
\text { optimized feature set }\end{array}$ & difference \\
\hline Sprouted wheat & $79.10 \%$ & $82.00 \%$ & $\mathbf{+ 2 . 9 0 \%}$ \\
\hline Broken wheat & $82.80 \%$ & $86.80 \%$ & $\mathbf{+ 4 . 0 0 \%}$ \\
\hline Durum wheat & $91.60 \%$ & $94.40 \%$ & $\mathbf{+ 2 . 8 0} \%$ \\
\hline Wheat damaged by pests & $80.60 \%$ & $85.00 \%$ & $\mathbf{+ 4 . 4 0 \%}$ \\
\hline Oats & $96.50 \%$ & $96.20 \%$ & $\mathbf{- 0 . 3 0} \%$ \\
\hline Canola & $99.00 \%$ & $98.10 \%$ & $\mathbf{- 0 . 9 0 \%}$ \\
\hline Rye & $91.60 \%$ & $93.20 \%$ & $\mathbf{+ 1 . 6 0} \%$ \\
\hline Shrivelled wheat & $84.00 \%$ & $87.60 \%$ & $\mathbf{+ 3 . 6 0} \%$ \\
\hline Sunflower seeds & $98.10 \%$ & $97.80 \%$ & $\mathbf{- 0 . 3 0} \%$ \\
\hline Husks & $89.10 \%$ & $90.00 \%$ & $\mathbf{+ 0 . 9 0 \%}$ \\
\hline Stones & $95.40 \%$ & $96.20 \%$ & $\mathbf{+ 0 . 8 0} \%$ \\
\hline Weed seeds & $94.00 \%$ & $94.70 \%$ & $\mathbf{+ 0 . 7 0 \%}$ \\
\hline Other contaminations & $77.50 \%$ & $80.80 \%$ & $\mathbf{+ 3 . 3 0 \%}$ \\
\hline Flawless wheat & $82.10 \%$ & $86.90 \%$ & $\mathbf{+ 4 . 8 0 \%}$ \\
\hline total recognition rate & $\mathbf{8 8 . 9 2 \%}$ & $\mathbf{9 0 . 9 5 \%}$ & $\mathbf{+ 2 . 0 3 \%}$ \\
\hline
\end{tabular}

\section{Recognition rates of the final test}

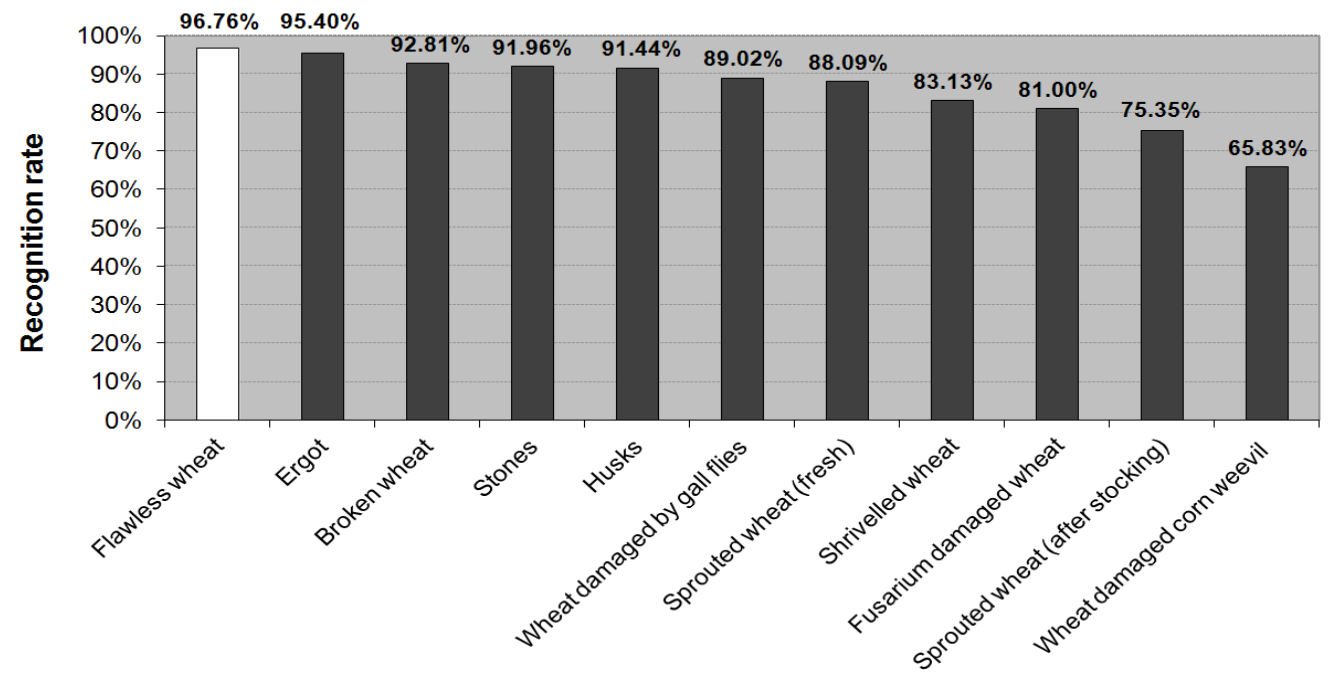

Fig. 10: Final test results with the material from different crop years

After this optimization, the dataset has been expanded with toxic ergot and fusarium-damaged wheat and a final training dataset with nearly 40.000 objects as well as optimized features and optimized SVM parameters (regularization parameter $\mathrm{Nu}$ and parameter Gamma of the radial basis function kernel) were used to create a classifier for practical testing. It was demonstrated that the whole system consisting of automatic sample separation, single kernel imaging and 
classification is able to achieve a high accuracy (Fig.10). Therefore, sample material with known composition from different crop years, also from later years than the material used for training, has been analysed in a comprehensive test.

\subsection{Classifier optimization}

After selecting the most discriminative features, there is the problem of choosing some parameters of the classifier. Support vector machines and neural networks turned out to be the most powerful classifiers at present. Classifiers with no parameters like K-nearest-neighbor and Naive Bayes are less powerful. Free parameters of classifiers affect the classification performance significantly. A common method to choose the free parameters of SVM is a grid search scheme. By giving the example of classifying mineral aggregates, the effectiveness of this method should be illustrated. One specific characteristic of this problem is the highly imbalanced dataset. There are some rare minerals with very few sample objects. A specialized separation device, described in [12] was used to separate the objects sized from $8-16 \mathrm{~mm}^{2}$ as preparation for an accurate image capturing during these experiments.

The SVM classifier [13] with the radial basis function kernel (rbf) was used for object classification. The kernel parameter Gamma and the regularization parameter $\mathrm{Nu}$ for the training of the SVM need to be chosen beforehand. To find an optimal parameter set, the mentioned grid search method in combination with 3 -fold crossvalidation on the training dataset with nearly 16.000 objects was applied.

The position of the grid points in the interval [0, MaxNu, Gamma] is calculated according to the following formula:

$$
\operatorname{Pos}_{N u, \text { Gamma }}=\frac{\operatorname{Max}_{N u, \text { Gamma }}}{2^{i-n+1}} \quad \text { with } \quad 0<=\mathrm{i}<=9 \text { and } \mathrm{n}=10
$$

The formula leaves an exponential characteristic for the distribution of the nodes. The interval width grows with increasing values for $\mathrm{Nu}$ and Gamma. As expected, the optimal values of both parameters are small. To handle the imbalanced dataset, the predictive power is measured in terms of the balanced error rate (BER). This measure is defined as the average of the error rates in each class. For many recognition problems with a natural material, the influence of Gamma is much higher than that of $\mathrm{Nu}$. Nu controls the training set error as well as the number of training vectors which become support vectors and, thus, affects the decision border to become more complex. With growing Gamma, the influence of each of these support vectors on the decision border is growing. To cover the high intra-class-variance in the dataset, many relevant training vectors are needed with each relatively little but equally distributed influence among them on the decision border. This results in a low value for Gamma and a medium scale value for $\mathrm{Nu}$ (Fig.11). If the parameter $\mathrm{Nu}$ is increased significantly over 0.005 , a decrease of the performance could also be monitored. 
Machine Learning and Applications: An International Journal (MLAIJ) Vol.3, No.3, September 2016

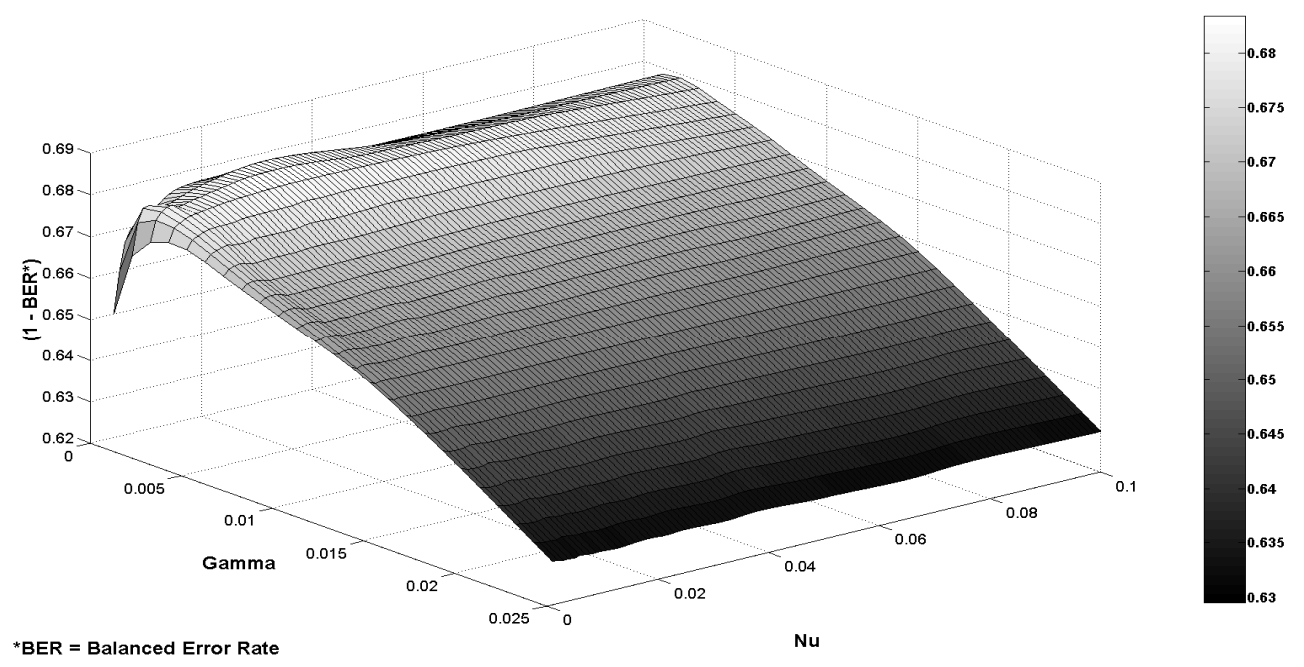

Fig. 11: Performance of the SVM as a function of the parameters Nu and Gamma

For the final system test, 16 different mineral aggregates have to be combined into four groups according to their hazard potential for the production of concrete (group 1 - non-hazardous; group 2 till 4 - hazardous). The test showed that mineral aggregates can be assigned to the groups with an accuracy of nearly $90 \%$.

Table 2: Results of the classification of mineral aggregates into the four relevant groups

\begin{tabular}{|c|c|c|c|c|c|c|c|}
\hline & & \multicolumn{4}{|c|}{ classified pebble category } & \multirow[b]{2}{*}{\begin{tabular}{|l|} 
recognition \\
rate per class \\
{$[\%]$} \\
\end{tabular}} & \multirow[b]{2}{*}{\begin{tabular}{|l|} 
total \\
recognition \\
rate [\%] \\
\end{tabular}} \\
\hline & $\begin{array}{l}\text { real pebble } \\
\text { category }\end{array}$ & $\begin{array}{l}(1-9) \\
\text { Group 1 } \\
\end{array}$ & \begin{tabular}{|c|}
$\begin{array}{l}\sum_{13}(10,11,12, \\
\text { Group 2 }\end{array}$ \\
\end{tabular} & $\begin{array}{l}\sum(14) \\
\text { Group } 3 \\
\end{array}$ & \begin{tabular}{|l|}
$(15,16)$ \\
Group 4 \\
\end{tabular} & & \\
\hline \multirow{10}{*}{$\begin{array}{c}\text { Group } \\
1\end{array}$} & 1 quartz & 181 & 3 & 0 & 2 & \multirow[b]{10}{*}{91.1} & \multirow{16}{*}{88.1} \\
\hline & 2 chert & 221 & 0 & 1 & 10 & & \\
\hline & 3 quartzite & 203 & 14 & 0 & 4 & & \\
\hline & 4 graywacke & 155 & 2 & 0 & 4 & & \\
\hline & $\begin{array}{l}5 \text { other paleozoic } \\
\text { sediments }\end{array}$ & 192 & 10 & 0 & 7 & & \\
\hline & $\begin{array}{l}6 \text { sandstone, } \\
\text { siltstone, claystone }\end{array}$ & 171 & 28 & 0 & 0 & & \\
\hline & 7 limestone & 198 & 35 & 0 & 2 & & \\
\hline & 8 volcanic rocks & 184 & 8 & 1 & 16 & & \\
\hline & $\begin{array}{l}9 \text { basement rocks } \\
\text { (e.g. granite, gneiss) }\end{array}$ & 193 & 9 & 0 & 5 & & \\
\hline & 10 dense flint & 213 & 5 & 0 & 21 & & \\
\hline \multirow{3}{*}{$\begin{array}{l}\text { Group } \\
2\end{array}$} & 11 porous flint & 15 & 72 & 1 & 2 & \multirow[b]{3}{*}{71.3} & \\
\hline & $\begin{array}{l}12 \text { light and porous } \\
\text { limestone and } \\
\text { marlstone }\end{array}$ & 21 & 68 & 0 & 0 & & \\
\hline & $\begin{array}{l}13 \text { friable } \\
\text { sedimentary rocks }\end{array}$ & 50 & 98 & 0 & 7 & & \\
\hline $\begin{array}{c}\text { Group } \\
\mathbf{3} \\
\end{array}$ & $\begin{array}{l}14 \text { brown coal, } \\
\text { xylit, carbonized } \\
\text { wood }\end{array}$ & 1 & 3 & 39 & 4 & 83.0 & \\
\hline \multirow{2}{*}{$\begin{array}{c}\text { Group } \\
4\end{array}$} & \multirow{2}{*}{$\begin{array}{l}15 \text { brown iron ore } \\
16 \text { pyrite, marcasite }\end{array}$} & 13 & 7 & 2 & 141 & \multirow[b]{2}{*}{86.2} & \\
\hline & & 6 & 0 & 1 & 40 & & \\
\hline
\end{tabular}




\section{VISUALISATION OF DATASET COMPLEXITY}

The two given recognition tasks and the results obtained show a high complexity which is due to the specific characteristic of natural materials. A good way for visualising the underlying complexity of the recognition problem is the displaying of the first three principal components after principal component analysis (PCA) [14] on the given dataset. The PCA allows the visualisation of the given data based on a reduction of the relevant information by using only the first three principal components with the highest variance. The goal of the PCA is the approximation of the $\mathrm{p}$ features by a smaller number of meaningful linear combinations (principal components).

A PCA and a visualisation of object clusters in the feature space was realised with Matlab ${ }^{\circledR}$ for obtaining a better understanding of the two given recognition problems and the separability of the classes by using the feature vectors with all calculated features. The visualisation of the first three principal components is always connected with a loss of information for complex highdimensional data. Nevertheless, it allows the comparison of the underlying complexity between different recognition problems, especially in consideration of the calculated total variance of the first three principle components. The first three principal components of selected object classes for grain analysis and the four grouped classes of the mineral aggregates recognition problem are visualized in Fig.12 and Fig.13.

The visualization of the object clusters of both recognition tasks shows that the automatic classification of natural materials is a highly complex and sophisticated challenge. Several grain classes like flawless wheat, sprouted wheat, and wheat infected by fusarium show an overlap [15]. Some classes are well defined, and their distance to each other is greater than the dimension of the clusters (for example: flawless wheat / ergot, flawless wheat / oat). On closer inspection of the cluster forms it is visible that the classes wheat damaged by fusarium and flawless wheat are relatively smoothed and strongly limited spherical or ellipsoid clusters. Oat and ergot form classes of long dimensional ellipsoid-clusters. However, the classes toxic and nontoxic weed seeds constitute irregular expanded clusters without a criterion for homogeneity, because these classes have a high intra-class variability based on the great amount of weeds seed subspecies employed. Here, the calculated total variance of the first three principal components is only $41.1 \%$.

In Fig.12, no homogeneous and well-defined clusters can be found for the classes of mineral aggregates. All four superordinate classes (groups 1 to 4 ) show a far-reaching overlap so that no class boundaries in this space can be found. Here, the calculated total variance of the first three principal components is $44.3 \%$ but the clusters of the given data cannot be separated in this feature space.

In summary, it becomes clear that the recognition task of the given classes of mineral aggregates is more complex than the recognition task of the given classes of grain. 
Machine Learning and Applications: An International Journal (MLAIJ) Vol.3, No.3, September 2016

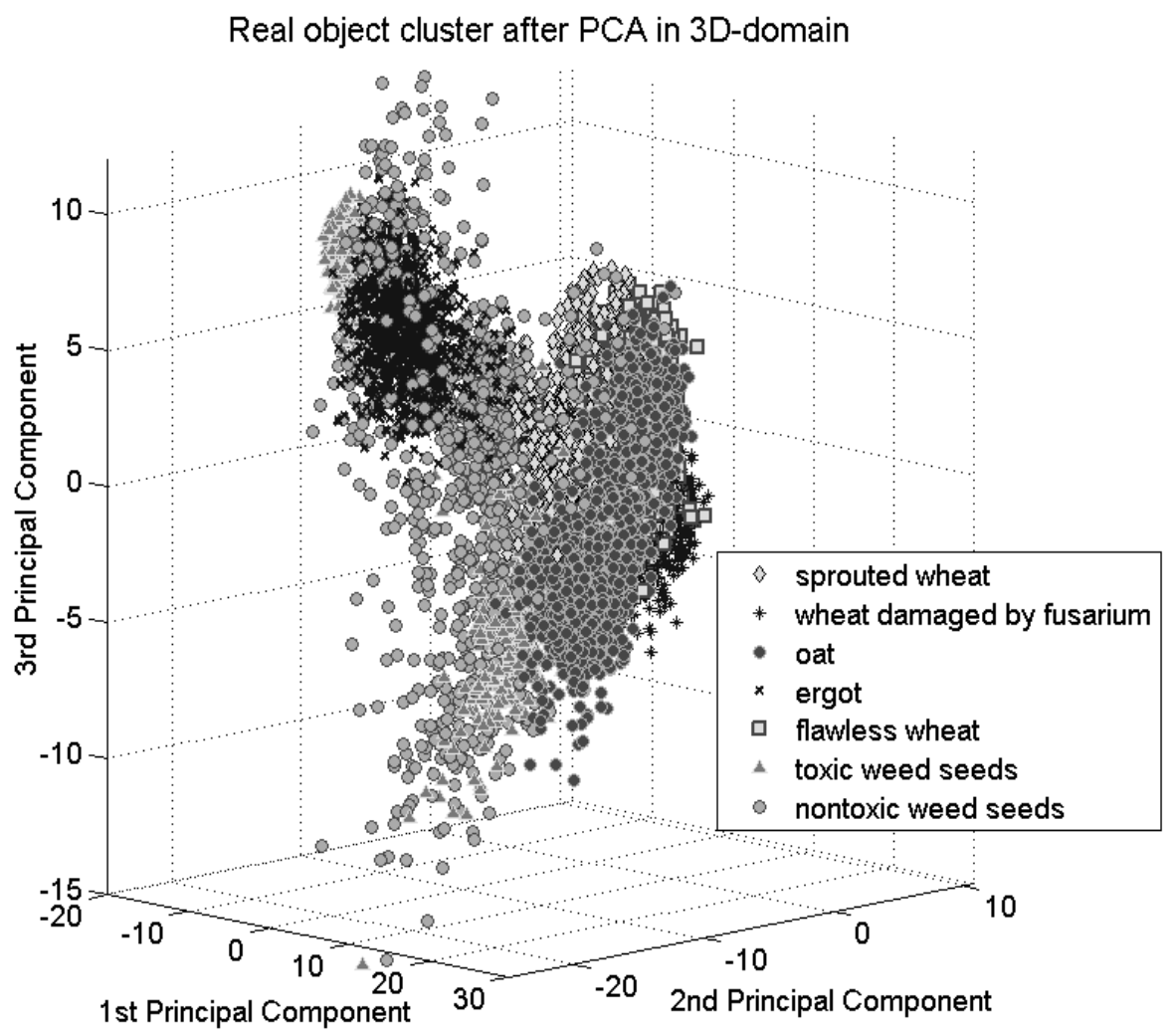

Fig. 12: Classes of grain - real cluster representation after PCA in 3D-domain

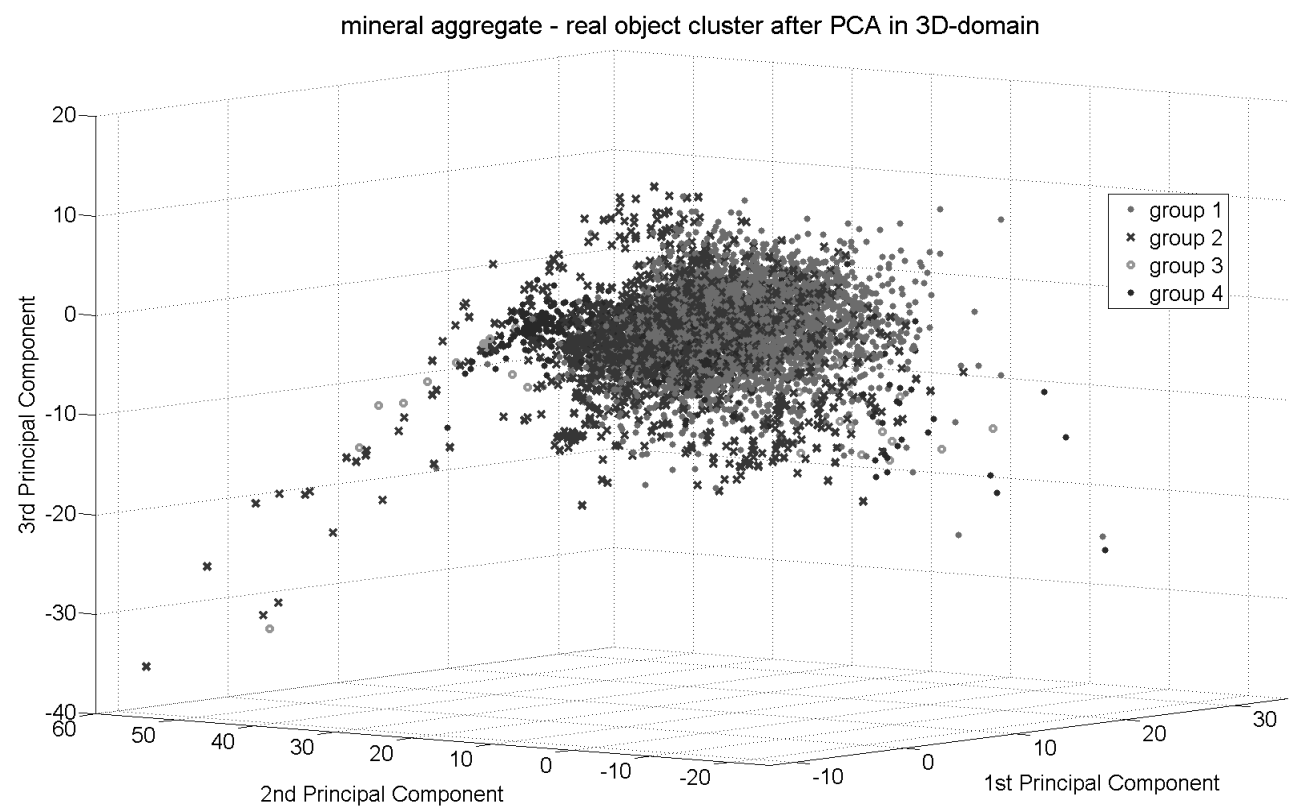

Fig. 13: Mineral aggregates - real cluster representation after PCA in 3D-domain 


\section{RESULTS}

The automatic recognition of miscellaneous materials, especially natural materials, is always a challenging task. Recent advantages in the development of new classification algorithms like support vector machines, random forest and neural networks now make it possible to solve many of these problems. The first algorithmic step of every visual recognition task is the segmentation. In our paper, we presented a solution to separate highly overlapping and touching objects in the overall image into single object images. Efficient segmentation and feature extraction in real-time makes inline processing for many sorting and observation tasks possible. Field-programmable gate arrays and graphic processing units can be used to enhance the processing speed of the feature extraction, if necessary.

One fact should be kept in mind: The chance for overfitting is huge with the high phenotypic variability of natural material. Feature selection and the right choice of the model parameters can help to reduce this effect. The parameter optimization for the problems mentioned has been done on a high-performance desktop computer. For a higher amount of data, a broader domain or a higher number of parameters, distributed computing and genetic algorithms can be used to speed up the calculation. The principal component analysis is a tool to evaluate the separability of the different classes in the feature space. Such investigations should be the first step in the development of a recognition system to get an impression of the feasibility.

This article presented some problems of automatic recognition with image processing systems and offered possible solutions. With modern software tools for image processing and classifier design it is possible to solve these problems in an efficient way. The robustness of automatic recognition systems for natural materials can only be conclusively assessed after a long period of practical testing. Especially natural materials are often affected by certain instable conditions, e. g. the effects of weather on the annual crop. The results of the presented research activities indicated that it is possible to automate the process of designing image-based recognition systems at least for low and medium complex recognition problems. Such a system is currently under development. The recognition of defects on metallic surfaces is chosen as field of application to show the principle feasibility. Some first results are published in [16] and [17].

\section{REFERENCES}

[1] Color Sorting Buhler Sortex Limited Great Britain, URL: www.sortex.com, invocation: March 2015

[2] Mineral Sorting Mogensen Sorting Machines, URL: www.mogensen.de/en/color-sorting-miningmineral-sorting-machines.htm, invocation: March 2016

[3] H. Drobny e.a.: Ein neues digitales Imaging-System zur genauen Erfassung von GetreideQualitätsparametern (A new imaging system for the registration of grain quality parameters), Du Pont de Nemours (Deutschland) GmbH, 2008

[4] L. Lepistö e.a.: Combining Classifiers in Rock Image Classification, URL: www.cs.tut.fi/ avisa/digger/Publications/ACIVS2004_Lepisto.pdf, invocation: March 2016

[5] S. Bauer, D. Mann, F. P. León, Applicability of hyperspectral fluorescence imaging for mineral sorting, Proc. Conf. Opt. Character. Mater. 2, p. 205-214, 2015

[6] S. Lerm: Objektsegmentierung von kompaktem Schüttgut für die technische Erkennung (Object Segmentation in Compact Bulk Material for Automatic Recognition), dissertation, Ilmenau University of Technology, 2013

[7] I. Guyon, A. Elisseeff: Feature extraction: foundations and applications, Berlin, Springer Verlag, 2006

[8] T. M. Mitchell: Machine Learning, The Mc-Graw-Hill Companies Inc, 1997

[9] Halcon, Reference Manual - Halcon 11.0.5, MVTec Software GmbH, URL: www.mvtec.com, invocation: June 2014 
Machine Learning and Applications: An International Journal (MLAIJ) Vol.3, No.3, September 2016

[10] D. Garten: Einfluss von Bildaufnahme und Bildmerkmalen auf die Erkennungsguete bei der automatischen Besatzanalyse von Brotweizen (Influence of image acquisition and image features on the recognition rate for the automatical Besatz analysis of wheat), dissertation, Ilmenau University of Technology, 2011

[11] K. Anding; P. Brückner; M. Dambon; D. Garten: Measuring Wheat Quality. IN: Journal for Vision Systems Design, Volume 15, Issue 6, 2010

[12] K. Anding, D. Garten, E. Linß, G. Pieper: Klassifikation mineralischer Baurohstoffe aus Lockergesteinslagerstätten mittels Bildverarbeitung und maschinellem Lernen (Classification of mineral aggregates from loose rock deposits with image processing and machine learning), 16th Workshop of the German Color Group, 2010

[13] P.-H. Chen, C. -J. Lin and B. Scholkopf, A tutorial on nu-support vector machines, 2003

[14] W. J. Krzanowski: Principles of Multivariate Analysis, Rev. ed. Oxford University Press, Oxford 2000

[15] K. Anding: Automatisierte Qualitätssicherung von Getreide mit überwachten Lernverfahren in der Bildverarbeitung (Automatic quality assurance of grain using supervised machine learning in image processing), dissertation, Ilmenau University of Technology, 2010

[16] D. Garten, K. Anding: Inspection of surface finish and dimensional accuracy of metal parts. Fraunhofer IOSB KCM SpectroNet Collaboration Forum, Karlsruhe, 2015

[17] K.V. Trambitskiy, K. Anding, G.A. Polte, D. Garten, V.M. Musalimov: Out-of-focus region segmentation of 2D surface images with the use of texture features, Scientific and Technical Journal of Information Technologies, Mechanics and Optics, 2015, vol. 15, no. 5, pp. 796-802.

\section{Authors}

Daniel Garten studied computer science at the University of Applied Sciences in Schmalkalden from 2003 to 2007. After graduation, he gained his doctor's degree in the knowledge field of industrial image processing from the Ilmenau University of Technology in 2011. He works as a project engineer and deputy division manager at the Society for Production Engineering and Development (Gesellschaft fuer Fertigungstechnik und Entwicklung Schmalkalden e. V.) in the field of optical measurement and recognition in tool industry. He was awarded the GFaI Young Talent Award 2011 (GFaI - Gesellschaft zur Förderung angewandter Informatik) and the VIU Young Talent Award 2013 (VIU - Verband innovativer Unternehmen e. V.).

Katharina Anding studied mechatronics at the Ilmenau University of Technology from 2001 to 2005. After getting her diploma degree, she gained her doctor's degree in the knowledge field of industrial image processing from the Ilmenau University of Technology in 2010. She works as a project leader in the area of image processing and machine learning. In 2011, she was awarded the STIFT Young Talent Award (STIFT Stiftung für Technologie, Innovation und Forschung). She teaches image processing and machine learning at the Ilmenau University of Technology.

Steffen Lerm studied mechatronics at Ilmenau University of Technology from 2000 to 2007. After graduating he became a research assistant. In 2013 he finished his $\mathrm{PhD}$ in the knowledge field of industrial image processing at Ilmenau University of Technology. During his time as a research assistant, he has dealt with several problems in digital image processing and spectroscopy.
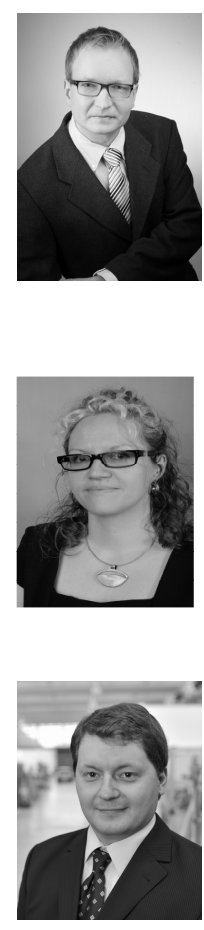